\title{
BIENESTAR Y DESARROLLO: EVOLUCIÓN DE DOS CONCEPTOS ASOCIADOS AL BIEN VIVIR
}

\author{
Well-Being and development: Evolution of two concepts associated \\ with the good living
}

Ricardo López Salazar*

\section{RESUMEN}

En este artículo se analiza desde un punto de vista reflexivo las diferentes teorías sobre el Estado de bienestar y desarrollo. Metodológicamente se utilizó el análisis documental, consistente en identificar las principales corrientes teóricas sobre el bienestar, utilizando la perspectiva desarrollada por Titmuss (1987) Mkandawire (2005) y el desarrollo, desde las visiones de Boisier (1999), Vázquez-Barquero (2005) así como Thorbecke (2007). Se identifica que ambos, son conceptos en constante construcción, asociados al devenir de una sociedad específica con sus problemas y retos. Por lo que, como constructos sociales complementarios, persiguen el bien vivir de las personas. Por lo que, en esencia, el desarrollo significa qué y cuáles características asociadas a la vida, debe de poseer una persona o familia para poder considerar vive bien en un contexto determinado. En otras palabras, bienestar y desarrollo son dos conceptos indisociables y seguramente persistirán como puntales en la agenda de los Estados-nación y de la sociedad civil organizada.

Palabras clave: bienestar, desarrollo, políticas a favor de los pobres, universalismo, focalización

RECIBIDO: Noviembre 2018

ACEPTADO: Enero 2019

\section{ABSTRACT}

\footnotetext{
* Profesor-Investigador de Tiempo Completo Titular C en la Universidad Autónoma de Ciudad Juárez, División Multidisciplinaria Nuevo Casas Grandes. Doctor en Ciencias con especialidad en Desarrollo Regional por el Centro de Investigación en Alimentación y Desarrollo, CIAD, A.C. México. Ha publicado diversos artículos sobre pobreza y políticas públicas en revistas especializadas de circulación nacional e internacional, y dictaminados artículos en revistas nacionales e internacionales. Miembro del Sistema Nacional de Investigadores (SNI), nivel I, CONACYT. Segundo Lugar Premio FAOCLACSO (2018) "Investigación en innovación de políticas públicas para la Seguridad alimentaria y nutricional", categoría artículo publicado. Chihuahua, México. Correo electrónico: ricardo.lopez@uacj.mx
} ORCID: https://orcid.org/0000-0002-0769-5330 
In this article, the different theories about the welfare state and development are analyzed from a reflective point of view. Methodologically, the documentary analysis was used, consisting of identifying the main theoretical currents on well-being, using the perspective developed by Titmuss (1987) Mkandawire (2005) and the development, from the visions of Boisier (1999), Vázquez-Barquero (2005) as well as Thorbecke (2007). It is identified that both are concepts in constant construction, associated with the evolution of a specific society with its problems and challenges. Therefore, as complementary social constructs, they pursue the well-being of people. Therefore, in essence, development means what and what characteristics associated with life, must have a person or family to be able to consider living well in a determined context. In other words, well-being and development are two inseparable concepts and will surely persist as mainstays in the agenda of nation-states and organized civil society.

Key words: welfare, development, pro-poor policies, universalism, targeting.

\section{Introducción}

El vivir dignamente, desde siempre ha sido una de las metas trazadas por las personas a lo largo de su vida. Sin embargo, al realizar un ejercicio estrictamente cronológico, el bienestar a grosso modo inició en el lenguaje gubernamental, académico y en organizaciones como los sindicatos, desde la finalización de la Segunda Guerra Mundial. Diversos autores como Titmuss(1987) señalan al bienestar como resultado directo de la transformación del Estado, en particular de su crecimiento el cual favoreció la expansión y oferta de servicios públicos básicos como la salud, educación, vivienda, empleo, entre otros. Al parecer, el bienestar es un concepto en constante construcción, sobre todo por su naturaleza ligada al desarrollo concreto de una sociedad específica. En este menester, el desarrollo emerge como un complemento al bienestar, en el sentido de su asociación en la misma dirección.

Clarificando lo anterior, el bienestar puede ser entendido como los aspectos vinculantes, concretos para que las personas consideren a su estado como bien vivir, por su parte, el desarrollo son los facilitadores de lo primero, como la correcta 
aplicación de políticas de fomento al empleo, el respeto al estado de derecho, el acceso a los sistemas de salud y educación, por citar algunos.

Con base en lo anterior, este artículo tiene como objetivo, analizar dichos conceptos tomando como punto de partida la construcción del Estado de bienestar y su vinculación con el desarrollo. Para cumplir dicho cometido, en el análisis documental, identificamos las principales corrientes sobre el bienestar y desarrollo, y las principales propuestas de ambos conceptos relacionadas con el bien vivir. El documento se estructura de la siguiente manera: en la primera parte se revisa, describe y analiza el Estado de bienestar. En la segunda, se ocupa del desarrollo y su evolución desde los 50's hasta nuestra época. Finalmente se presentan conclusiones sobre los temas abordados.

\section{El Estado de Bienestar}

Las políticas públicas desde la época de la posguerra han jugado un papel clave en el desarrollo de la sociedad. La emergencia de conceptos como el Estado de bienestar, la seguridad social para todos, los servicios sociales y de salud al alcance de las masas, fueron uno de los principales motores del crecimiento de la economía mundial durante la segunda mitad del siglo pasado, Lampmam(1984). Dicha época, comenzó con un Estado impulsor de la actividad económica en la época de la segunda revolución industrial, posterior a la finalización de la Segunda Guerra Mundial y terminó con el gobierno tomando como responsabilidad el proveer de los servicios más elementales a la población.

De acuerdo con Barr (1992) el estado de bienestar comenzó sobre la consigna de generar mayores oportunidades para la población, y para ello, generalmente, existen cuatro grandes áreas a considerar tanto de manera privada como pública para impulsarlo. En primer lugar, está la generación de mercados laborales competitivos y bien remunerados, para proveer a las personas ingresos suficientes para utilizarlos en la compra de bienes y servicios. El segundo, estriba con la capacidad de las personas para 


\section{Ricardo López Salazar}

Telos Vol. 21, No. 2 (2019). 288-312

generar su bienestar de manera privada, ya sea mediante el ahorro o la compra de seguros médicos o educativos. El tercero, consiste en la búsqueda de bienestar al interior y exterior de los integrantes de la familia. En el cuarto, el Estado juega un papel primordial, al asegurar o por lo menos generar las condiciones propicias para el desarrollo efectivo de las tres áreas descritas anteriormente, asimismo, el Estado puede implementar políticas impositivas orientadas a financiar o favorecer el mercado laboral y el bienestar privado de las familias

El objetivo central del Estado de bienestar como su nombre lo indica, consiste en la generación de un nivel adecuado de vida para las personas, bajo el entendimiento de la inclusión de elementos básicos sociales como: protección social, acceso a los servicios de salud y educación, y la posibilidad de encontrar un empleo estable y con remuneración digna.

Por supuesto, la persecución de tal objetivo involucra necesariamente la alineación y consecución de otros más específicos, entre los más destacados en palabras de Barr (1992):

1.- Eficiencia (involucra aspectos como la eficiencia macroeconómica y microeconómica).

2.- Mejorar los estándares de vida (incluye la contabilización de cuántos pobres existen, a cuántos es necesario apoyar, cuántos recursos habrán de ocupars, etc.).

3.- Reducción de la desigualdad (transfiriendo recursos a los pobres e implementando programas de empleo y generación de ingresos).

4.-Integración social (incluye la prestación de servicios básicos como el acceso a salud y educación).

5.- Factibilidad administrativa (referida a una administración sencilla y propicia para favorecer a la actividad económica y también alejada de prácticas abusivas como la corrupción). 
La implementación del Estado de bienestar involucra todo un complejo aparato de soporte para su implementación. La implementación de dicho aparato, durante la época posterior a la posguerra involucró grandes erogaciones de recursos económicos, materiales y humanos Bergin (1999). No obstante, tal derroche fue posible, gracias a cuatro fenómenos presentes en el contexto de posguerra. En primer lugar, el crecimiento poblacional experimentado en casi todos los países desarrollados y en desarrollo. En segundo lugar, asociado al crecimiento poblacional, aumentaron las necesidades de la población en lo referente a los servicios básicos, como el acceso a servicios educativos, de salud, de vivienda, entre otros, necesitando muchos recursos para desarrollarlos. En tercer lugar, la reconstrucción de Europa y los abundantes recursos provenientes del plan Marshall favoreció el desarrollo de la actividad económica tanto en Estados Unidos, como en los países de mediana industrialización. Por último, la consolidación del capitalismo como el modo de producción dominante favoreció la creación del Estado-Nación con amplísimas fortalezas asociadas a la incipiente democracia en los países en desarrollo (Bergin, 1999).

\section{Universalismo vs Focalización del bienestar}

Como bien menciona Titmuss(1987), cualquier discusión relacionada con el Estado de bienestar y sus objetivos pasa por el establecimiento de la utilización del enfoque de proveer servicios universales para todos o de proveer dichos servicios de manera focalizada. En la misma línea, el autor anterior señala como el establecimiento de servicios universales para toda la población, al menos para el caso de la Gran Bretaña convergió sobre la premisa de asegurar correspondencia entre derechos ciudadanos y la adquisición de los servicios elementales como salud y educación.

Entonces, queda más o menos clara la noción del universalismo parte del reconocimiento de: "todos los ciudadanos por el sólo hecho de pertenecer a una sociedad merecen tener acceso a los servicios sociales mínimos". Por supuesto, dentro del "universalismo" conviven una serie de visiones, retoricas, retos y modelos más 
complejos y trascendentes al simple postulado de "servicios sociales para todos por todos" (Valencia Lomelí et al., 2010).

Particularizando el análisis hacia algunos sistemas universales, como el Alemán y Norteamericano, éstos nacieron bajo la premisa de proteger a los trabajadores del sector industrial durante la época de la gran depresión, y, en segundo lugar, como una forma de control de masas y protección de las élites ante el descontento ciudadano debido a las dificilísimas condiciones económicas. Desde la óptica de Baldwin (1989), el universalismo y el desarrollo de las políticas sociales obedecieron a una reconfiguración histórica para favorecer la integración de la clase trabajadora más humilde como una forma de reivindicación del Estado, en parte, porque el Estado de bienestar en su inicio excluyó a dichas clases. En palabras de Titmuss (1987), el universalismo es más bien una respuesta del Estado hacia la construcción de ciudadanía.

Por el contrario, desde la visión de Kildal y Kuhnle (2002), la selectividad o focalización involucra a las personas con acceso a los servicios sociales deben poseer ciertas "características deseables" y deben ser corroboradas mediante una serie de evaluaciones o de escrutinio por parte de las autoridades. En este punto, Mkandawire (2005), señala que la diferencia central entre ambos es la cobertura, así, en el universalismo toda la población disfruta de los beneficios sociales como un derecho básico, en tanto el enfoque selectivo, la elegibilidad para los beneficios sociales está sujeta a alguna forma de comprobación previa de medios de vida para determinar a los "verdaderos merecedores". Sin embargo, tampoco es posible afirmar la no ejecución de acciones selectivas de localización o focalización de los recursos de manera discrecional en el universalismo.

Es decir, los regímenes de políticas no son casi nunca enteramente universales ni netamente selectivos, tienden más bien a ubicarse en algún lugar entre los dos extremos de un "continuo", y con frecuencia son híbridos; sin embargo, su ubicación 
en este continuo puede resultar decisiva para determinar las posibilidades de subsistencia de cada persona y para caracterizar el orden social.

Ahora, surge una pregunta natural ¿por qué surge la visión o el enfoque selectivo o focalizado en cuanto al acceso de las personas a los servicios sociales? Posiblemente una respuesta robusta se oriente a situar como causa principal a los recursos necesarios a emplear para proveer de servicios sociales a todas las personas. En la misma línea, Gilbert (2004), apunta el "proveer servicios para todos" estructuralmente acarrea grandes erogaciones de recursos al Estado, por lo cual, surge como una opción la "parcialización de los servicios" o la discriminación en cuanto al acceso de los mismos. En este sentido, el Estado convive bajo la presión de cómo hacer frente a las crecientes demandas de su población en términos de erogación financiera y una de las salidas más socorridas ha sido precisamente, el enfoque selectivo o focalizado de la acción social, como medida para "racionar" los recursos.

Aunque el razonamiento anterior, puede ser más o menos acertado, incluso lo es aún más si éste lo circunscribimos a un aspecto de carácter estructural como es el cambio de enfoque económico implementado en las últimas cuatro décadas, fincado en eliminar de manera progresiva los subsidios para evitar fallas de mercado. De este modo, el trasfondo de la parcialización y con ello la selectividad en cuanto a la provisión de servicios sociales obedece a eliminar los "free riders", es decir, aquellos beneficiarios de servicios públicos en parte por sus ingresos o estatus social pueden pagar servicios privados, pero gracias a los subsidios o a la existencia de servicios públicos de calidad, prefieren la utilización de los últimos.

Dejando de lado las razones y causas favorecedoras de la utilización del enfoque universalista o focalizado y selectivo, la cuestión central consiste en conocer cuál de los dos ofrece mejores resultados en cuanto a la generación de seguridad social y combate a la pobreza. Según Mkandawire (2005), a priori la focalización debería ofrecer ventajas en la identificación de los pobres o de las personas susceptibles para recibir los beneficios de las políticas sociales, sin embargo, en muchas ocasiones la 


\section{Ricardo López Salazar}

Telos Vol. 21, No. 2 (2019). 288-312

identificación de los beneficiarios involucra el desarrollo de una serie de sistemas sofisticados igual o más onerosos comparativamente hablando con la estructura administrativa soporte de la perspectiva universalista. Sin embargo, lo anterior, no responde la cuestión central, porque se abordan procedimientos, estructura o componentes en lugar de resultados. Es decir, en el fondo es insuficiente tratar de conocer cuál método enfoque o aproximación de política es más oneroso, sino cuál de ellos rinde mejores resultados, en otras, palabras la cuestión estriba en determinar qué enfoque conduce a la gente a vivir mejor.

Para Mkandawire (2005), el universalismo genera mejores resultados en cuanto a la reducción de la pobreza, sobre todo, en función de incorporar más aspectos a la sola utilización del concepto de pobreza absoluta como se hace en la perspectiva de la focalización. De acuerdo con el mismo autor, el universalismo ha otorgado una serie de experiencias positivas respecto al combate a la pobreza, mientras, la focalización ha resultado en algunas ocasiones en deficiente selección de beneficiarios, desperdicio de recursos y ha creado un sistema o engranaje de intereses políticos en contraposición a su visión social para lo que fueron originalmente concebidos.

Sin embargo, de acuerdo con Edillon (2017), los programas de transferencias de efectivo condicionadas, son efectivos en la reducción de la pobreza, al menos para el caso de Filipinas donde logró una reducción de la misma (pequeña) a partir de un programa de tipo condicionado. En relación a esta discusión, Nelson (2007), analizó los efectos de diversos programas de seguridad social (seguro contra el desempleo, seguro de salud, pensiones para personas adultas, etc.) para un conjunto de dieciocho países desarrollados, y encontró, pese al avance a nivel académico y gubernamental de las propuestas hacia programas condicionados como forma de atender las necesidades sociales, el universalismo, en general, aún es mejor para lograr seguridad social.

No obstante, debemos señalar la inexistencia de una respuesta única, conciliadora y afirmativa porque depende en demasía de las condiciones específicas del 
Bienestar y desarrollo: evolución de dos conceptos asociados al bien vivir

territorio o país ejecutor de las políticas y de otras variables exógenas como el crecimiento económico o la correcta aplicación del estado de derecho. Aunque es preciso matizar, los programas condicionados suelen perder eficacia cuando la población beneficiaria deja de serlo, entonces, la premisa inicial sustentante de la focalización como es el racionamiento de los recursos, origina otra cuestión como ¿cuánto tiempo es necesario o suficiente para una persona o familia a partir de los recursos obtenidos por programas de transferencias de efectivo condicionadas, salga de la situación o nivel de pobreza para dejar de ser beneficiaria? O en otro nivel, ¿Cómo "evitar" el crecimiento en la cobertura de los programas focalizados deriven en erogaciones de recursos similares a las demandadas por programas de tipo universal? Ambas interrogantes involucran un extenso debate superior al objetivo del presente escrito, pero sin lugar a dudas, sintetiza parte de la discusión sobre los programas universalistas y los focalizados.

\section{Políticas a favor de los pobres.}

¿Es posible dirigir el crecimiento hacia los pobres?, o en otro nivel, ¿Qué tipo de estrategias y acciones son necesarias implementar para vincular al crecimiento económico y la redistribución de ingreso con los estratos sociales más desfavorecidos? Con base en estas dos interrogantes, existe toda una corriente de políticas públicas conocidas como Pro-Poor (en nomenclatura inglesa, a continuación, a favor de los pobres) éstas buscan el desarrollo e implementación de acciones dirigidas a la reducción y el abatimiento de la pobreza. El razonamiento central de las políticas a favor de los pobres recae en la disyuntiva de: el crecimiento económico por sí mismo no genera una reducción progresiva de la pobreza, la marginación y la desigualdad. Incluso en algunas ocasiones éstas tienden a exacerbarse aun cuando algunos países han experimentado procesos sostenidos de crecimiento económico como bien señala Pasha (2003). Por tanto, para Kakwani (2000), el crecimiento económico es una condición necesaria más no suficiente para el combate y reducción de la pobreza, y por ende, esto plantea y 


\section{Ricardo López Salazar}

Telos Vol. 21, No. 2 (2019). 288-312

exhibe la necesidad de generar acciones congruentes con los problemas y las oportunidades de las regiones y localidades respecto al combate a la pobreza.

Respecto a por qué el crecimiento económico no necesariamente deriva en reducción de la pobreza y la desigualdad, autores como Kakwani (2000) y Ravallion (2004), observan en trabajos como el de Dollar y Kraay (2002), como el crecimiento económico sostenido refleja una disminución en los niveles de pobreza al menos en los datos agregados, a nivel particular, en otras ocasiones el crecimiento económico provoca segmentación y especialización en los mercados laborales, los cuales reducen la oportunidad de los pobres (generalmente poseedores de menos cualificaciones técnicas o educativas) para emplearse, provocando una profundización de la desigualdad.

El mismo Kakwani (2000), explica como la relación entre el crecimiento económico y la pobreza es por sí misma compleja, y dicha complejidad está determinada por los niveles y cambios en la desigualdad y la pobreza. Es decir, generalmente las políticas orientadas hacia los pobres consisten en favorecer el crecimiento económico y la reducción de la desigualdad y la pobreza, no obstante, tal definición deja muchas aristas y dudas sobre su medición y más aún sobre su implementación. En palabras del mismo autor, una definición más sólida de qué es una política a favor de los pobres, en primer lugar, categoriza entre relativo y absoluto el crecimiento y su impacto entre los pobres. Dicho de otra manera, el aspecto relativo refiere cuando el crecimiento económico beneficia mayormente a los pobres comparados con los no pobres, y por tanto, ello implica una reducción relativa en la desigualdad. El aspecto absoluto refiere cuando los pobres a causa del crecimiento, obtienen mayores beneficios comparado con las personas no pobres.

Respecto al efecto absoluto en la reducción de la pobreza a través de políticas a favor de los pobres, Ravallion (2004), visualiza esto como un proceso en el cual los pobres resultan mayormente beneficiados con el crecimiento económico, cuando existe 
Bienestar y desarrollo: evolución de dos conceptos asociados al bien vivir

la conjunción de diversos aspectos como: una adecuada combinación de políticas públicas y reformas impulsoras del crecimiento y la integración de los pobres en dicho proceso. No obstante, para el citado autor, no existe una "receta única" de políticas y reformas, o de estrategias y acciones definitivas para reducir la desigualdad y la pobreza, pero, al menos cabe la posibilidad de aprender de los casos específicos a nivel nacional e internacional y sus resultados de éxito.

Algunos resultados prometedores en cuanto a la aplicación de tales políticas, implementadas en el África Sub-sahariana, y en Asia han sido reportadas. En esa línea, Pasha (2003), identifica como las políticas a favor de los pobres han otorgado una serie de lecciones a considerar en el momento de su aplicación, resaltan las siguientes:

1.- Identificar a los sectores donde funcionan las políticas (como la agricultura)

2.-Identificar áreas susceptibles en donde implementarse (generalmente áreas en subdesarrollo).

3.- Identificar los factores de producción internos (incluso a las personas sin habilidades).

4.- Identificar las salidas (por ejemplo, el consumo de alimentos).

En suma, las políticas a favor de los pobres ofrecen una visión interesante respecto a la relación entre tres conceptos claves de la economía y la sociedad, como es el crecimiento económico, la pobreza y la desigualdad, poniendo en el centro del debate la posibilidad de cómo el crecimiento realmente contribuye a reducir la pobreza y la desigualdad. Entonces, el concepto posee una serie de fortalezas innegables, destacando a sobremanera la sencillez de su postulado central. No obstante, carece de un desarrollo homogéneo, debido precisamente a su aplicación heterogénea, es decir, las situaciones, los contextos, los actores y los orígenes de la falta de crecimiento, altas tasas de pobreza y desigualdad generalmente obedecen a aspectos específicos a las regiones, y a los países. Sin embargo, la visión de las políticas orientadas hacia el beneficio de los pobres deja lecciones positivas, en el sentido de la posibilidad de generar crecimiento económico incluyente y equitativo, aunque para ello cabe identificar plenamente los 


\section{Ricardo López Salazar}

Telos Vol. 21, No. 2 (2019). 288-312

sectores, los insumos, las capacidades y las áreas puente entre el crecimiento, la reducción de la pobreza y la desigualdad.

De tal suerte, el primer reto consiste en la realización de un diagnóstico adecuado, y posteriormente canalizar las acciones de políticas o reformas vinculantes de las tres variables anteriormente citadas. Entonces, el segundo reto estriba en cómo lograr la sostenibilidad en el tiempo de las tasas de crecimiento del PIB y asociado a ello, la generación y desarrollo de una red de seguridad social efectiva para todos con énfasis en políticas redistributivas a nivel social, redundante en el cumplimiento de la ley y la integración de los pobres al proceso de crecimiento. Sin lo anterior, probablemente el crecimiento económico derive en la ampliación de la desigualdad lo cual reduce el efecto de las políticas a favor de los pobres.

\section{El Desarrollo}

Como parte de la complejidad relacionada con la definición de qué es el bienestar, han emergido diversos conceptos orientados a coadyuvar y complementar dicha tarea. En ese sentido, el concepto de desarrollo es uno de los más relevantes cuando analizamos de manera particular al bienestar mismo. En otras palabras, el desarrollo sirve de enlace entre el bienestar objetivo y subjetivo para materializarse en la persona y sus condiciones de vida. No obstante, previo a profundizar sobre el vínculo entre bienestar y desarrollo es pertinente enfocarse más a detalle en el desarrollo, porque a evolucionado con el paso del tiempo y bien merece ser analizado con mayor prestancia.

Dicho lo anterior, es preciso notar como el desarrollo es un concepto, en palabras de Boisier(1999), bastante complejo en su caracterización porque es resultado de los nuevos reacomodos derivados de la Segunda Guerra Mundial emparejado a un nuevo orden económico, político y social. Tal complejidad, racae en el tránsito por diversas etapas de "maduración" y sobre todo de integración de diversos aspectos 


\section{Bienestar y desarrollo: evolución de dos conceptos asociados al bien vivir}

complementarios al bienestar, lo anterior es posible identificarlo mediante un simple análisis de las corrientes económicas dominantes previo a la Segunda Guerra Mundial y subsecuentemente a ésta. Así, por ejemplo, las principales corrientes económicas como el Keynesianismo identificaban al Estado a través de sus diversos instrumentos (e.g. política económica, política fiscal, política monetaria, por citar algunos) poseía la irreductible obligación de estimular el crecimiento económico, fundamentalmente el del Producto Interno Bruto (PIB) como objetivo primario del desarrollo. Particularmente a partir de 1945 (fin de la Segunda Guerra Mundial) el pensamiento keynesiano fue el paradigma a seguir en detrimento de otras escuelas y pensamientos como el liberalismo. Aunque en esencia, y a riesgo de ser repetitivo, el pensamiento keynesiano dominó la escena económica y política a nivel internacional hasta mediados de los años 80’s, lógicamente fue sujeto de diversas interpretaciones, cada una de ellas orientadas a estimular el crecimiento económico bajo el designio primario del Estado (favoreciendo el surgimiento del Estado de Bienestar).

La primera interpretación clara del modelo consistió en estimular el crecimiento de las actividades industriales (idea sustentada en la noción de los efectos multiplicadores de Keynes) en contrapartida de las actividades agrícolas y pecuarias. También conocido como modelo de economía cerrada, mediante la cual una actividad moderna (industria) era impulsada y por el contrario, "castigaba" a la otra actividad de corte arcaico (agricultura), acompañada de medidas de protección hacia los productos industriales fabricados internamente en detrimento de los de origen externo. Dicha etapa, categorizada como Industrial Sustitutivo de Importaciones (ISI) en buena parte de los países en vías de desarrollo. Naturalmente, debemos cuestionar ¿qué implicaciones generó dicha interpretación del paradigma keynesiano para el desarrollo? La respuesta es simple en su elucubración, pero evidentemente compleja en su interpretación, debido a dos aspectos centrales. El primero, radica en la premisa de estimular al sector industrial la economía crecería, y con ello, paulatinamente a través del TrickleDown generaría un efecto positivo para el resto del sistema y por ende a 
través de un efecto filtro, los beneficios llegarían hacia todas las personas. El segundo, conectado estrechamente con el primero, en el apartado de las personas, porque al crecer la economía a través del PIB, crecería el ingreso personal y con ello el acceso a una mayor cantidad de bienes y servicios elevarían el bienestar. Podemos notar como las primeras nociones del desarrollo, encauzaron la visión economicista del crecimiento y su vínculo lineal con el ingreso y su posterior efecto en el bienestar, sin considerar la posibilidad del surgimiento de distorsiones e imperfecciones de mercado generalmente suscitadas en el marco del funcionamiento del sistema económico de un país.

$\mathrm{Al}$ inicio de la década de los 60`s y con ello la emergencia de la llamada "revolución verde" revitalizó el papel de la agricultura como motor de crecimiento económico. De tal suerte, en diversos países, especialmente en los latinoamericanos surge el llamado dualismo económico, centrado en la promoción a la industria y la reincorporación de la agricultura como un sector importante pero no al mismo nivel del primero. Sin embargo, al menos, reconoció su rol en la creación de empleos, fortalecimiento del ingreso y por añadidura al crecimiento de las economías. De acuerdo con Thorbecke (2007), en dicho periodo, el Estado, además de ocuparse de impulsar el crecimiento económico, también incorporó a sus actividades programáticas la implementación de políticas de corte social como las relacionadas con los servicios de salud, educación, acceso a la alimentación (controlando y subsidiando los precios de los alimentos) por citar algunos. De esta manera, el concepto de desarrollo no solamente centra su objeto de manera exclusiva en el crecimiento del ingreso personal, al incorporar "nociones" del capital humano como el nivel educativo y la salud, si bien, la formalización de este último concepto ocurrirá décadas más adelante.

A pesar del dualismo económico imperante durante la década en cuestión, comenzó un análisis discriminante al interior de los dos sectores en la búsqueda de cuál de los subsectores y ramas de los mismos retornarían mayores rendimientos a la inversión. Es decir, el criterio de invertir en la industria y/o agricultura, o en ambos, se 


\section{Bienestar y desarrollo: evolución de dos conceptos asociados al bien vivir}

sustituye por otro más específico, dirigido a identificar qué rama de la industria (e.g. química, metálica, extracción de petróleo y gas) o el cultivo de cereales, hortalizas o de otros cultivos, serían más rentables y por ende susceptibles de ser apoyados. En la lógica anterior, primaba un aspecto crucial, además del retorno económico de la misma inversión y la expectativa de generación de empleos, la contraparte, el desempleo, comenzó a situarse como un fenómeno complejo de atacar, en parte, por las masivas migraciones del campo a la ciudad ocurridas durante la década previa, coadyuvando a estigmatizar al campo como poco importante.

Así, emergieron una serie de problemas socioeconómicos ocasionados por la toma de decisiones basadas en el pragmatismo económico, como el desempleo urbano, el crecimiento sin control de las ciudades, y la consecuente generación de clases sociales menos favorecidas en cuestión de servicios públicos básicos dando lugar a las periferias urbanas y la acentuación de la desigualdad.

Los desafíos vislumbrados en los 60`s fueron más profundos durante los 70’s, en particular el desempleo y la informalidad catalizadores de la explosión de la pobreza urbana. En el recuento de los hechos, el desarrollo ya no puede circunscribirse solamente al crecimiento del PIB, porque no garantizaba su transmisión hacia el bolsillo de las personas de manera directamente proporcional, además, el descarte previo del campo como un sector estratégico provocó el surgimiento de los fenómenos ya comentados, en este punto, cabe replantear la interrogante esgrimida por Boisier (1999) ¿Desarrollo, de qué estamos hablando? Y su respuesta en esencia debe enmarcarse en la lucha contra la desigualdad, la marginación y la pobreza, a través del fomento de políticas de empleo, de recuperación de los espacios rurales y el fortalecimiento en el acceso a los servicios públicos como la salud, la educación, el agua, el alcantarillado, y la energía eléctrica para toda la población.

Los 80 `s presenta, quizá uno de los cambios más importantes en el paradigma y doctrina económica, sintéticamente incluyó el adelgazamiento del Estado y por ende su alejamiento a través de la privatización de las principales actividades económicas 
poseídas por éste como comunicaciones telecomunicaciones, energía, finanzas, por mencionar las más relevantes. Dicho paradigma ha sido identificado como neoliberalismo, al retomar las ideas anteriormente propulsadas por los economistas liberales de principios de siglo como la apertura económica y la reducción de aranceles, eliminación de las cuotas a la importación y la desregulación de sectores clave, pero aderezados por la concepción de un nuevo proceso de intensificación a escala planetaria de los mismos. Por ende, fenómenos como la globalización, la desregulación financiera y comercial han sido la nueva ruta crítica para retomar el sendero de crecimiento que, cabe decir, entró en clara fase de agotamiento con el modelo keynesiano.

Si en los 70`s el combate a la pobreza y a la desigualdad, sobre todo en las grandes urbes fue una condición sine qua non para medir la efectividad del modelo y de los propios Estados-nación, en los 80's, dos palabras: "ajuste y equilibrio" dominaron el escenario gubernamental. Curiosamente desde el ámbito gubernamental, la noción de ser un agente causante de más problemas a las soluciones conseguidas, permeó de forma profunda como doctrina de acción en los Estados. Lo anterior, reforzó el alejamiento del mismo de las actividades económicas y sustantivas al bienestar, con lo que en definitiva la visión Keynesiana dejó de ser el paradigma dominante para darle paso al liberalismo económico.

A pesar del citado alejamiento del Estado y los imperativos ajustes en el modelo de desarrollo, surgió el concepto de desarrollo humano, posicionándose como la contraparte al "ajuste y equilibrio". De tal suerte, el Estado omnipresente y ejecutor de manera discrecional, dónde, cuándo, cuánto y en qué invertir, se sustituyó por la visión del desarrollo humano, al posicionar como la inversión más redituable recae en las personas sobre las actividades y los sectores. Es decir, bien podemos señalar a dicho enfoque (desarrollo humano) como la inspiración detrás de los programas focalizados de transferencias económicas condicionadas implementados en México (bajo los nombres de Progresa, Oportunidades y recientemente, Prospera). 
De tal suerte que, invertir en las personas en aras de incrementar sus posibilidades de incorporación a la "sociedad productiva" a través del ejercicio pleno de sus libertades, ideas esencialmente propulsadas por Amartya Sen, sin lugar a dudas, coadyuvó a sortear la parálisis exhibida por los Estados-nación, en el ejercicio de políticas públicas dirigidas a fomentar el bienestar y desarrollo en la década de los 80’s. No obstante, es preciso puntualizar como las políticas públicas, sobre todo las de carácter social, comenzaron a tomar un camino, hasta la fecha sin regreso, focalizándose hacia grupos considerados como vulnerables, eliminando de facto la persecución del Estado de Bienestar por todos y para todos, por una visión ciertamente discriminadora de quien y quiénes con base en ciertos parámetros serán susceptibles de ser apoyados por el Estado. En sustancia, el desarrollo humano, intenta posicionarse como un concepto encaminado a responder la interrogante planteada con anterioridad (¿Desarrollo, de qué estamos hablando?), y a partir de allí, diseñar estrategias concretas para superar los obstáculos suscitados en el combate a la pobreza, la desigualdad y la marginación.

En esencia, el Desarrollo Humano, ha sido ampliamente aceptado, en parte por su operacionalización relativamente sencilla y porque combina tres elementos para evaluar el progreso de los países en la materia: el Producto Interno Bruto (PIB) por habitante, la salud y la educación; cada uno es ponderado al mismo nivel. De acuerdo con López-Calva (2004), debido a su simplicidad, y requisición de información, generalmente disponible para su construcción, lo convierte en el punto de referencia más utilizado para realizar comparaciones internacionales e incluso muchos países han adoptado los índices de desarrollo humano como instrumento de política y como indicador del éxito o fracaso de sus políticas nacionales. No obstante, también existen diversas críticas hacia el desarrollo humano, sobre todo, en la parte normativa del mismo, y han surgido nuevas aportaciones posicionadas en la era del posmodernismo y el posdesarrollo criticando abiertamente el establecimiento de criterios arbitrarios de 


\section{Ricardo López Salazar}

Telos Vol. 21, No. 2 (2019). 288-312

estándares de vida a cumplir para señalar si una sociedad debe considerar como desarrollada o subdesarrollada.

Si para Amartya Sen y la ONU, las personas como receptores de las acciones del Estado y de los demás agentes interventores en el funcionamiento de un país son los sujetos de análisis para medir al desarrollo. Otros autores dirigieron sus baterías hacia los transmisores de dichas acciones, particularmente, al finalizar la década de los 80's, y sobre todo al principio de los 90`s, autores como Meyer-Stamer (1995) y North (1990), identificaron a los ajustes realizados previamente en la escala macroeconómica y sobre todo del modelo y paradigma económico como acertados, éstos no eran suficientes para convertirse en los conductores hacia una nueva senda de crecimiento con estabilidad, equidad y bienestar para las personas.

La causa principal inferida por los autores en cuestión estribó en la paradoja del funcionamiento de un sistema económico. Lo anterior, sintetizado idealmente en dos ámbitos, microeconómico y macroeconómico, sin lugar a dudas era necesario incorporar un eslabón entre ellos para potenciarlos y dirigirlos hacia la misma senda. Igualmente, para Meyer-Stamer (1995), dicho vínculo debe ser identificado como las políticas meso, las cuales deberían de convertirse en una suerte de amalgama entre los agentes económicos micro y macroeconómicos. Por su parte, North (1990) dirige su centro de análisis hacia las instituciones a las cuales señala como los soportes de las iniciativas, acciones, estrategias, políticas y programas de cualquier gobierno o Estado. Es decir, North (1990), retoma en parte el argumento de la burocracia esgrimido por Webber un siglo atrás, pero particularizando a ésta como un obstáculo a sortear para la correcta implementación de las reformas de los 80’s para generar resultados tangibles en los 90`s y reducir la galopante desigualdad en la distribución del ingreso.

En otra línea de análisis, autores como Vázquez-Barquero (2005) o Bosier (1999), coinciden en el funcionamiento correcto de la macroeconomía, la microeconomía, de las políticas intermedias y sobre todo de las instituciones, son 
altamente relevantes para disminuir la desigualdad y la pobreza, presentan una interrogante sumamente pertinente: ¿qué sucede con los actores locales en el proceso de desarrollo? Dicha interrogante, aunque retoma la noción de desarrollo local esgrimida desde principios del siglo pasado por Marshall y su idea de los distritos industriales como fuente de desarrollo, ésta permaneció inactiva en las principales corrientes académicas de la época ante la emergencia del keynesianismo y del gran monopolio ejercido por el Estado en la economía desde los 20`s hasta los 70’s.

Sin embargo, con el devenir del siglo y el desmoronamiento de la economía planificada y la emergencia del mercado como el vértice central sobre el cual giraría una nueva época sostenida de bienestar económico y ante los nuevos problemas generados por este modelo, la idea del desarrollo local comienza a tomar fuerza desde diversos círculos académicos para enfrentar estos retos.

Sin embargo, existen notables controversias acerca del desarrollo local y su emergencia como corriente teórica de importancia. Desde la visión de Boisier (1999), por desarrollo local debe entenderse al proceso capilar, local, endógeno, continuo o discontinuo sobre el territorio, y a diferencia del crecimiento puede ser inducido desde arriba o desde abajo, además responde a particularidades concretas sólo atribuibles a una realidad específica. También señala la coexistencia de múltiples visiones de desarrollo local como desarrollo endógeno, desarrollo económico local, desarrollo descentralizado, no abonan a finalizar la confusión del mismo y, por tanto, derivan en la dificultad de su implantación como un concepto homogéneo.

Por otro lado, Vázquez-Barquero (2005), identifica al desarrollo local como un proceso que incluye la concertación de una serie de acciones a través de actores locales enfocadas a desarrollar sus capacidades. Dichas capacidades, indudablemente deben tomar como iniciativa central incrementar el bienestar de las personas. De esta definición podemos inferir como el desarrollo no es un proceso exclusivo de un actor en particular, ni tampoco polariza a los actores públicos y privados, por el contrario, 


\section{Ricardo López Salazar}

Telos Vol. 21, No. 2 (2019). 288-312

intenta generar convergencias y uniones para favorecer la emergencia de iniciativas concretas a una localidad o a un territorio.

Entonces, el desarrollo local en sustancia y acción implica la emergencia de actores públicos y privados encaminados a desarrollar las potencialidades internas del territorio y/o localidad en aras de incrementar el bienestar de las personas. La simple aproximación anterior es por sí misma más compleja, y habríamos de poner sobre la mesa una serie de aspectos más profundos, como los grados de responsabilidad de los actores públicos y privados en el proceso, los tipos de coordinación, el rol y la importancia de las instituciones locales, por mencionar algunas. Sin embargo, clarificar lo anterior, sobrepasa los límites del presente trabajo, y por tanto, nos abocaremos a otros aspectos del desarrollo.

Con el inicio del nuevo milenio, el debate acerca del desarrollo ha sido dominado por tres fenómenos: globalización, desigualdad y pobreza. Motivo de profundas discusiones y estudios ha sido sujeta la globalización y su efecto vinculante en la exacerbación y/o disminución de la desigualdad y la pobreza. Desde la esfera gubernamental, aspectos como el buen gobierno, la incorporación de la sociedad en la toma de decisiones (gobernanza), combate a la corrupción y fortalecimiento institucional representado por los crecientes pasos en relación a transparentar las funciones del Estado, han tomado un lugar de primer orden en la agenda pública. Habiendo realizado un recuento (no exhaustivo) de las diversas teorías y enfoques del desarrollo surgidas a partir de la finalización de la Segunda Guerra Mundial es adecuado cerrar la sección con lo siguiente.

Es innegable como el concepto de desarrollo ha experimentado diversas transformaciones con el devenir de los años (véase tabla 1), sin embargo, en esencia, se debe entender como aquellos elementos vinculados a una sociedad específica, y asociados de manera objetiva al bien vivir de una persona o familia. En otras palabras, bienestar y desarrollo son dos conceptos indisociables, y seguramente persistirán como 
puntales en la agenda de los Estados-nación y de la sociedad civil organizada. No obstante, es imperativo mencionar a la contraparte del bienestar y desarrollo (pobreza/desigualdad) lejos de adentrarse a un proceso de franca reducción, a nivel mundial, muestran una tendencia preocupante y lacerante, donde la concentración del ingreso, exhibe niveles históricos con alrededor de $11 \%$ de la población más rica concentrando el 82\% del ingreso mundial (OXFAM, 2018). Adicionalmente, la sobrevivencia en países con menos de un dólar y el limitado acceso a satisfactores básicos como el agua, la energía eléctrica, la salud y la educación aparecen como retos perennes. De tal suerte, cabría esperar, durante los años venideros, nuevas rutas de acción en la escena pública, académica y social, orientadas a la consecución de mayor bienestar y desarrollo para todos. El presente y futuro lo demandan.

Tabla 1. Evolución de las teorías del desarrollo (1950-2000)

\begin{tabular}{|c|c|c|c|c|c|}
\hline Año & Objetivos & $\begin{array}{ll}\text { Políticas } & \mathbf{y} \\
\text { estrategias } & \end{array}$ & $\begin{array}{ll}\text { Teorías } & \text { e } \\
\text { Hipótesis } & \end{array}$ & $\begin{array}{l}\text { Modelos } \quad y \\
\text { técnicas }\end{array}$ & $\begin{array}{l}\text { Sistemas de } \\
\text { medición }\end{array}$ \\
\hline 1950 & $\begin{array}{l}\text { Crecimiento } \\
\text { de PNB }\end{array}$ & $\begin{array}{l}\text { Sustitución de } \\
\text { importaciones, } \\
\text { industrialización, } \\
\text { inversión en } \\
\text { infraestructura. }\end{array}$ & \begin{tabular}{lr} 
"Big push". & \multicolumn{2}{r}{ Take Off y } \\
etapas & de \\
crecimiento. \\
Tesis esfuerzo \\
mínimo crítico. \\
Criterios \\
inversión de \\
agregada
\end{tabular} & $\begin{array}{l}\text { Harrod- } \\
\text { Domar }\end{array}$ & $\begin{array}{l}\text { Las cuentas } \\
\text { nacionales de } \\
\text { los ingresos. }\end{array}$ \\
\hline 1960 & $\begin{array}{l}\text { Crecimiento } \\
\text { del PNB } \\
\text { Balance de } \\
\text { pagos de } \\
\text { equilibrio } \\
\text { Empleo }\end{array}$ & $\begin{array}{l}\text { Los precios de } \\
\text { juste. } \\
\text { Crecimiento } \\
\text { equilibrado entre } \\
\text { agricultura e la } \\
\text { industria. } \\
\text { Promoción de las } \\
\text { exportaciones. } \\
\text { Ayuda externa. } \\
\text { Integración } \\
\text { regional. } \\
\text { Reformas } \\
\text { fiscales. Planes } \\
\text { sectoriales }\end{array}$ & $\begin{array}{l}\text { Dualismo } \\
\text { económico. } \\
\text { Vínculos } \\
\text { intersectoriales. } \\
\text { Protección } \\
\text { efectiva. Capital } \\
\text { humano. Precios } \\
\text { sombra } \\
\text { Elección de la } \\
\text { técnica. } \\
\text { Patrones de } \\
\text { crecimiento. El } \\
\text { papel de la } \\
\text { agricultura }\end{array}$ & $\begin{array}{l}\text { Input- } \\
\text { Output } \\
\text { Equilibrio } \\
\text { general } \\
\text { simple } \\
\text { Programaci } \\
\text { ón lineal }\end{array}$ & $\begin{array}{l}\text { Cuentas } \\
\text { nacionales de } \\
\text { ingresos } \\
\text { Input-Output } \\
\text { Censos de } \\
\text { empleo } \\
\text { Cuentas } \\
\text { Nacionales } \\
\text { Sociales }\end{array}$ \\
\hline
\end{tabular}




\begin{tabular}{|c|c|c|c|c|c|}
\hline 1970 & $\begin{array}{l}\text { Crecimiento } \\
\text { PNB } \\
\text { Empleo } \\
\text { Distribución } \\
\text { del ingreso } \\
\text { Reducción } \\
\text { pobreza } \\
\text { Equilibrio } \\
\text { extremo }\end{array}$ & $\begin{array}{l}\text { Desarrollo rural } \\
\text { integrado } \\
\text { Estrategias de } \\
\text { empleo integrado } \\
\text { Redistribución } \\
\text { con crecimiento } \\
\text { Reformista } \\
\text { Radical- } \\
\text { colectivista }\end{array}$ & $\begin{array}{l}\text { Enfoque del } \\
\text { paquete en zona } \\
\text { rural } \\
\text { Papel del sector } \\
\text { informal } \\
\text { La migración } \\
\text { rural-urbana } \\
\text { Tecnología } \\
\text { apropiada } \\
\text { Relación y } \\
\text { compromiso } \\
\text { entre } \\
\text { producción, } \\
\text { empleo, } \\
\text { distribución del } \\
\text { ingreso } \\
\text { pobreza. y }\end{array}$ & $\begin{array}{l}\text { Teoría de la } \\
\text { dependencia } \\
\text { Objetivos } \\
\text { múltiples } \\
\text { Los } \\
\text { modelos } \\
\text { sectoriales }\end{array}$ & $\begin{array}{l}\text { Cuentas } \\
\text { nacionales de } \\
\text { ingresos } \\
\text { Input-Output } \\
\text { Censos de } \\
\text { empleo } \\
\text { Encuestas de } \\
\text { hogares } \\
\text { Encuesta rural } \\
\text { Encuestas } \\
\text { sector } \\
\text { informal } \\
\text { Información } \\
\text { demográfica }\end{array}$ \\
\hline 1980 & $\begin{array}{l}\text { Estabilizaci } \\
\text { ón. } \\
\text { Equilibrio } \\
\text { externo } \\
\text { (balanza de } \\
\text { pagos). } \\
\text { Equilibrio } \\
\text { interno } \\
\text { (presupuest } \\
\text { aria y } \\
\text { monetaria). } \\
\text { Ajuste estructural. } \\
\text { Eficiencia }\end{array}$ & $\begin{array}{l}\text { Estabilización y } \\
\text { ajuste estructural. } \\
\text { Orientación hacia } \\
\text { el exterior. } \\
\text { Dependencia de } \\
\text { los mercados. } \\
\text { Privatización. } \\
\text { Minimización del } \\
\text { papel del } \\
\text { gobierno. }\end{array}$ & $\begin{array}{l}\text { Crecimiento } \\
\text { endógeno } \\
\text { Enlace entre } \\
\text { comercio y } \\
\text { crecimiento } \\
\text { Interrelaciones } \\
\text { entre humanos } \\
\text { Transferencia de } \\
\text { capital y } \\
\text { tecnología } \\
\text { Nueva } \\
\text { economía } \\
\text { institucional } \\
\text { Dependencia de } \\
\text { los mercados }\end{array}$ & $\begin{array}{l}\text { Impacto de } \\
\text { las políticas } \\
\text { fiscales y } \\
\text { comerciales } \\
\text { Modelos de } \\
\text { EGC } \\
\text { aplicados a } \\
\text { ajuste } \\
\text { estructural }\end{array}$ & $\begin{array}{l}\text { Matriz de } \\
\text { contabilidad } \\
\text { social } \\
\text { Ingresos y } \\
\text { gastos a gran } \\
\text { escala } \\
\text { Encuestas de } \\
\text { hogares }\end{array}$ \\
\hline 1990 & $\begin{array}{l}\text { Ajuste } \\
\text { continuo } \\
\text { El buen } \\
\text { gobierno y } \\
\text { las } \\
\text { institucione } \\
\text { s } \\
\text { Resurgimie } \\
\text { nto de alivio } \\
\text { de pobreza } \\
\text { Moderar } \\
\text { efectos del } \\
\text { caso de Asia } \\
\text { Limitar el } \\
\text { capitalismo } \\
\text { global }\end{array}$ & $\begin{array}{l}\text { Estabilización y } \\
\text { ajuste estructural. } \\
\text { Orientación hacia } \\
\text { el exterior. } \\
\text { Dependencia de } \\
\text { los mercados. } \\
\text { Reducción de la } \\
\text { pobreza y mejora } \\
\text { de bienestar } \\
\text { socioeconómico. } \\
\text { Controles y } \\
\text { regulaciones } \\
\text { adecuados en la } \\
\text { crisis financiera. }\end{array}$ & $\begin{array}{l}\text { El papel de las } \\
\text { instituciones en } \\
\text { el desarrollo. } \\
\text { Funciones de los } \\
\text { mercados y } \\
\text { gobierno. } \\
\text { Economía de } \\
\text { corrupción. } \\
\text { Capital social } \\
\text { como } \\
\text { complemento a } \\
\text { capital humano. }\end{array}$ & $\begin{array}{l}\text { Fuentes de } \\
\text { crecimiento } \\
\text { Productivid } \\
\text { ad total de } \\
\text { los factores } \\
\text { Regresiones } \\
\text { de } \\
\text { crecimiento }\end{array}$ & $\begin{array}{l}\text { Matriz de } \\
\text { contabilidad } \\
\text { social } \\
\text { Ingresos y } \\
\text { gastos a gran } \\
\text { escala } \\
\text { Encuestas de } \\
\text { hogares } \\
\text { Encuestas de } \\
\text { medición } \\
\text { estándar } \\
\text { Encuestas de } \\
\text { múltiples años } \\
\text { Encuestas } \\
\text { demográficas } \\
\text { y de salud }\end{array}$ \\
\hline
\end{tabular}


Bienestar y desarrollo: evolución de dos conceptos asociados al bien vivir

\begin{tabular}{|c|c|c|c|c|c|}
\hline 2000 & $\begin{array}{l}\text { Ajuste } \\
\text { continuo } \\
\text { El buen } \\
\text { gobierno y } \\
\text { las } \\
\text { institucione } \\
\text { s } \\
\text { Resurgimie } \\
\text { nto de alivio } \\
\text { de pobreza } \\
\text { Moderar } \\
\text { efectos del } \\
\text { caso de Asia } \\
\text { Limitar el } \\
\text { capitalismo } \\
\text { global } \\
\text { Desarrollo } \\
\text { humano }\end{array}$ & $\begin{array}{l}\text { Globalización } \\
\text { como estrategia } \\
\text { de desarrollo, } \\
\text { Búsqueda de } \\
\text { crecimiento }\end{array}$ & $\begin{array}{l}\text { Economía } \\
\text { política del } \\
\text { desarrollo y el } \\
\text { papel de las } \\
\text { instituciones. } \\
\text { Nexo } \\
\text { crecimiento- } \\
\text { desigualdad- } \\
\text { pobreza. } \\
\text { Pobreza } \\
\text { multidimension } \\
\text { al. Equilibrios } \\
\text { múltiples }\end{array}$ & $\begin{array}{l}\text { Critica de } \\
\text { regresiones } \\
\text { de } \\
\text { crecimiento }\end{array}$ & $\begin{array}{l}\text { Banco de datos } \\
\text { Ingresos y } \\
\text { gastos a gran } \\
\text { escala } \\
\text { Encuestas de } \\
\text { hogares } \\
\text { Encuestas de } \\
\text { medición } \\
\text { estándar } \\
\text { Encuestas de } \\
\text { múltiples años } \\
\text { Encuestas } \\
\text { demográficas } \\
\text { y de salud }\end{array}$ \\
\hline
\end{tabular}

Fuente: García (2015) con base enThorbecke (2007).

\section{Conclusiones}

El bienestar y el desarrollo representan dos de las mayores aspiraciones del ser humano. Si bien, dichos conceptos han mutado a lo largo del tiempo para imbricarse en el contexto específico de una sociedad, en una suerte de actualización discursiva, pragmática y de contenido. El análisis del bienestar individual o social incluye el estudio de las condiciones determinantes de la calidad de vida tanto de los individuos como de los grupos sociales; en este sentido, el análisis de la calidad de vida de una población dada es el objeto de estudio de la teoría económica del bienestar, en palabras de Huesca et al., (2011). En este sentido, el concepto de bienestar está estrechamente asociado al de desarrollo porque el último puede ser concebido como un proceso en el cual los individuos al mejorar sus condiciones y calidad de vida expanden sus libertades reales en el seno de la sociedad (Huesca et al, 2011). Sin embargo, en los últimos años, la predominancia de la economía de mercado, asociada a la desregulación comercial y financiera han desplazado de las agendas gubernamentales, las discusiones sobre cómo lograr el vivir mejor. 
Desde la óptica del presente escrito, las nociones del bienestar y desarrollo, además de ser retomadas como elementos centrales en las agendas de política de cualquier gobierno intencionado de mejorar la calidad de vida de sus ciudadanos, deben de incorporar la premisa de construir programas y políticas desde abajo, diagnosticando problemas comunes a las sociedades y trazando acciones y soluciones de la mano de los ciudadanos. De no ser así, posiblemente repitamos el mismo patrón observado en los últimos cincuenta años, resumido en transformaciones conceptuales, cristalizadas en acciones generalmente incompletas, ineficientes y alejadas de su objetivo central de lograr, precisamente a través del tiempo, las personas sienten mayor bienestar y por ende viven mejor.

\section{Referencias Bibliográficas}

Baldwin, Peter (1989). The Scandinavian Origins of the Social Interpretation of the Welfare State. Comparative Studies in Society and History, Vol. 31, No. 1. U.K. (pp. 3-24).

Barr, Nicholas (1992). Economic theory and the welfare state: A survey and interpretation. Journal of Economic Literature. Volumen 30, No. 2. EE.UU. (Pp.741-803).

Bergin, Meg (1999). Hey Big Spender: Testing Alternate Theories on Variance in Welfare National Effort.Res Publica - Journal of Undergraduate Research, 1999, Volumen 3, No 1. EE.UU. (Pp.35-48).

Boisier, Sergio (1999). Desarrollo (local) ¿de qué estamos hablando?, en Estudios Sociales, Corporación de Promoción Universitaria. Chile.

Dollar, David, y Kraay, Aart (2002). Trade, Growth, and poverty. The economic Journal. Volumen 114 No. 493. Oxford, England. (Pp. 22-49).

García, Carlos (2015). Teorías del Desarrollo. Universidad Autónoma de Ciudad Juárez. México.

Gilbert, Neil. (2004). Transformation of the Welfare State: The Silent. Oxford University Press, England.

Edillon, Rose Mary (2017). Universalism vs targeted social policy: Philippines' experience in addressing the challenges facing the poor and disadvantaged and marginalized groups. Expert Group Meeting onEradicating Poverty, UN. EE.UU. 
Huesca Reynoso, Luis, Mario Camberos Castro y Cuahutémoc Calderón Villarreal. (2011). Bienestar y desarrollo en el siglo XXI. Editorial Plaza y Valdés, México.

Kakwani, Nanak. (2000). Pro-poor growth: concetps and measurement with country case estudies. International Poverty Centre, Australia.

Kildal, Nanna, y Stein Kuhnle. (2002). The Principle of Universalism: Tracing a Key Idea in the Scandinavian Welfare Model. Paper presented at the Ninth

International Congress of Basic Income, European Network. Geneva, Italy. Lampmam, Robert. (1984) Social Welfare Spending. Accounting for Changes from 1950 to 1978. Institute for Research on Poverty Policy Analysis Series. Emerald Group Publishing Limited. United Kingdom.

López-Calva Luis (2004). Macroeconomía y pobreza: lecciones desde Latinoamérica. Serie Financiamiento del Desarrollo. CEPAL-ONU. Chile. Meyer-Stamer, Jörg (1995). Micro-level innovations and competitiveness. World Development. Volume 23, Issue 1, Amsterdam, Netherlands. (Pp. 143-148). Mkandawire, Thandika (2005). Targeting and universalism in poverty reduction. Social Policy and Development Programme Paper, $\mathrm{N}^{\circ}$ 23, Instituto de Investigación de las Naciones Unidas para el Desarrollo, Social (UNRISD) Switzerland.

Nelson, Keneth (2007). Universalism versus targeting: The vulnerability of social insurance and means-tested minimum income protection in 18 countries, 19902002. International Social Security Review, Volume 60, Issue 1. P.p. 33-58. Blackwell Publishing. United Kingdom.

North, Douglass (1990). Institutions, institutional change and economic performance. Editorial: Cambridge UniversityPress.EE. UU.

OXFAM (2018). Premiar el trabajo, no la riqueza. Informe internacional, OXFAM. United Kingdom.

Pasha, Hafiz. (2003). Pro-Poor Policies. Paper prepared for the ICNRD conference. Regional Bureau for Asia and the Pacific, UNDP.EE.UU.

Ravallion, Martin (2004). Pro-poor growth: A primer. World Bank Policy Research Working Paper 3242. EE.UU.

Thorbecke, Erik (2007). The evolution of the development doctrine, 1950-2005, Paper presented at the WIDER conference on the future of development economics, Helsinki, Finland.

Titmuss, Richard (1987). Welfare state and welfare society. In The Philosophy of Welfare. London: Allen and Unwin, United Kingdom.

Valencia Lomelí, Enrique y FoustRodriguez, David (2010). ¿Es pertinente pensar hoy en el universalismo en México? enValenciaLomelí, Enrique. Perspectivas del Universalismo en México. ITESO Amaroma Ediciones. México.

Vázquez-Barquero, Antonio (2005). Las Nuevas Fuerzas del Desarrollo. Antonio Bosh Editor. España. 